Association for Information Systems

AIS Electronic Library (AISeL)

Wirtschaftsinformatik 2021 Proceedings

Track 2: General Track - Innovative, emerging and interdisciplinary topics

\title{
Towards loT standards interoperability: A tool-assisted approach
}

Laurell Popp

Julius-Maximilians-Universität Würzburg

Melanie Schaller

iNDTact $\mathrm{GmbH}$

Follow this and additional works at: https://aisel.aisnet.org/wi2021

Popp, Laurell and Schaller, Melanie, "Towards loT standards interoperability: A tool-assisted approach" (2021). Wirtschaftsinformatik 2021 Proceedings. 6.

https://aisel.aisnet.org/wi2021/YGeneralTrack/Track02/6

This material is brought to you by the Wirtschaftsinformatik at AIS Electronic Library (AISeL). It has been accepted for inclusion in Wirtschaftsinformatik 2021 Proceedings by an authorized administrator of AIS Electronic Library (AISeL). For more information, please contact elibrary@aisnet.org. 


\title{
Towards IoT standards interoperability: A tool-assisted approach
}

\author{
Laurell Popp ${ }^{1}$, Melanie Schaller ${ }^{2}$ \\ ${ }^{1}$ University of Würzburg, Chair of Business Management and Business Information Systems, \\ Würzburg, Germany \\ \{laurell.popp\}@uni-wuerzburg.de \\ 2 iNDTact GmbH, Würzburg, Germany \\ \{mschaller\}@indtact.de
}

\begin{abstract}
Internet of Things (IoT) applications and ecosystems rely on the integration of numerous sensors and devices. One of the challenges of integration is the broadness of standards and protocols used by the sensors. At the same time, systems generally only support a limited amount of protocols, essentially limiting the choice of sensors and devices for a given scenario. In this paper, we propose a research methodology for building a tool that acts as middleware between sensors and systems, translating standards while maintaining their respective advantages.
\end{abstract}

Keywords: Internet of Things, messaging protocols, standardization

\section{$1 \quad$ Introduction}

The IoT is understood as the concept of turning physical objects into smart objects by equipping them with computational intelligence and transmitters and connecting them to the internet [1]. Thus, IoT is not understood to be one specific technology, but rather as a concept which utilizes multiple technologies. One element of this concept is connectivity, which typically follows the TCP/IP reference model. As such, IoT connectivity typically consists of four layers: the link (e.g., ethernet), internet (e.g., IPv6), transport (e.g. TCP), and application (e.g. HTTP) layers [2, 3]. For IoT data exchange purposes, multiple messaging protocols, which are part of the application layer, are designed for several scenarios [4,5]. Each standard serves a different set of requirements, making them advantageous in bandwidth, latency, and security for different sets of applications [4]. In many cases, practitioners cannot choose sensors or systems according to their advantages but rather if they can communicate by supporting the same messaging protocols. On the other hand, users may have to resort to using the "lowest common denominator" protocol, thus eliminating the advantages of specific messaging protocols.

In this paper, we explore the possibility of removing this problem using Peffers et al. Design Science Research (DSR) methodology [6] to build a tool conversing various messaging protocols while maintaining their respective advantages. In this context, we 
especially pay attention to future technologies such as $5 \mathrm{G}$ mobile radio standards and seek to preserve its benefits such as speed and latency reduction. Therefore, this work is primarily beneficial for practitioners seeking to use the full advantages of messaging protocols without limiting sensor selection.

\section{Related Work}

To validate the problem and take note of the current state of research in the subject on hand, an exploratory, unstructured literature review was conducted, which is part of phase one of our proposed methodology (see next section).

Several publications analyzed IoT messaging standards in a structured manner. Jaikar and Iyer, as well as Naik, compared messaging standards by several factors, such as speed, latency, security, and transport protocols [4,5]. Several authors identified the need to unify IoT applications, platforms, and ecosystems, some even referring to them as "silos of proprietary systems" [7]. The approach taken varies by use case and typically tries to enable interoperability between IoT platforms, architectures, ecosystems, messaging standards, or applications on a theoretical or practical level. Iglesias-Urkia and Casado-Mansilla integrated the CoAP messaging protocol and the theoretical standard IEC 61850 in the context of smart grids, thus mapping existing standards to a specific set of requirements laid out by a use case and the theoretical standard itself [8]. Kovacs et al. proposed an architecture for semantic interoperability using a limited set of international standards [9]. Desai et al. developed a semantic translation gateway for CoAP, MQTT, and HTTP protocols using a proxy architecture [7]. For sensing and actuation purposes, Yun et al. developed a platform that incorporates middleware programs for interoperability between the oneM2M standard and a set of other standards [10]. Zarko et al. developed a framework for the cooperation between IoT platforms on an organizational level by proposing so-called IoT platform federations and roaming IoT devices, where devices may switch between IoT platforms and interact with their respective resources [11]. Roth et al. proposed a framework for the interoperability between middleware platforms, thus enabling communication between smart environments [12]. Bandyopadhyay et al. identified and analyzed IoT reference architectures to build an understanding of the extend of interoperability of IoT standards [13]. Overall, these approaches are limited to a specific scenario or, in the case of Desai et al., to a limited set of messaging protocols. Our approach is not confined to a set of standards but instead tries to incorporate most of the currently used standards and thus enabling practitioners to be free in their choice of sensors or systems. In addition, none of the previous approaches paid attention to conserving specific advantages of certain standards, which is especially critical in cases where those advantages must be maintained, such as time-critical applications, for example, industrial wireless sensor networks [14]. 


\section{$3 \quad$ Methodology}

Our proposed methodology follows the DSR methodology introduced by Peffers et al. [6]. The goal of DSR in the field of business informatics is the creation and evaluation of artifacts of information technology to solve identified problems [15]. Thus, this article aims to propose an approach to address the issue at hand. In addition, we contribute to DSR in general by solving a practical problem. The DSR methodology proposed by Peffers et al. consists of six steps, which are shown in figure 1. Each step is supplemented by the specific approach and the generated output. The steps will be described briefly with our proposed approaches for each phase.

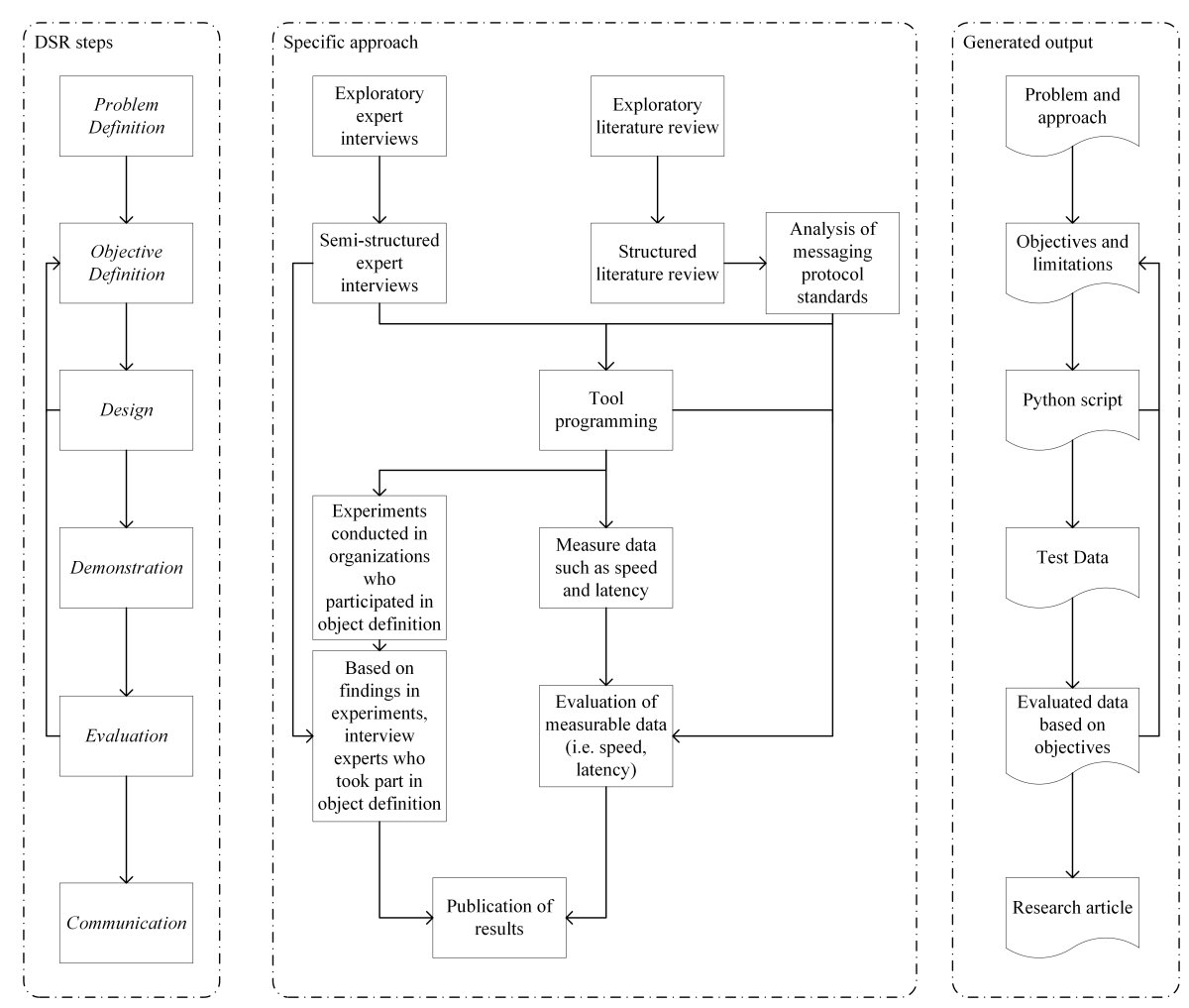

Figure 1. Proposed methodology following Peffers et al.

Problem Definition: At first, we define the problem by conducting a literature review and expert interviews, targeting experts (such as managing directors, CTOs, or CEOs) of companies in the field of sensor, system or infrastructure design in IoT environments. Both the expert interviews and the literature review serve as a concretization of the problem by identifying cases from both theory and practice, in which messaging protocols hindered the effective development of IoT infrastructures.

Objective Definition: Using the survey conducted in the first stage as a pre-test, we will be able to develop a standardized questionnaire for semi-structured expert 
interviews concerning usage of messaging protocol standards in practice following the methodology of Bell et al. [16]. By doing so, we intend to identify requirements (for examples, refer to chapter 4) from practice to define this research's objectives. The study targets approx. fifteen experts of companies in the field of sensor, system, or infrastructure design in IoT environments, thus reaching saturation of homogenous samples $[17,18]$. Furthermore, by conducting a subsequent, structured literature review, we identify and analyze currently used messaging standards. This step will yield a list of standards, their respective strengths, characteristics, and use cases. Consequently, we intend to determine which standards are to be supported by the artifact, thus defining further design objectives. By combining both approaches, we intend to define the objectives and limitations of the artifact to be developed.

Design: As a consequence of steps one and two, we develop a tool to convert the most commonly used messaging protocol standards by fulfilling the design objectives.

Demonstration: Due to the design artifact, we will perform experiments within the organizations which participated in the expert interviews of step one and two, demonstrating the feasibility of our solution. By conducting field tests with the designed tool, we will measure data such as speed and latency for a variety of predefined scenarios.

Evaluation: To evaluate our artifact, we test it against our defined objectives. Participating interview partners will be asked to take part in an evaluation interview. In addition, the measured data itself will be evaluated by comparing the results to the direct implementation of the respective standards.

Communication: The results will be published as a research article, and the code of the tool will be made available to the public as open-source code.

\section{Current Stage and Outlook}

First and foremost, we conducted an exploratory literature review, which is part of step one of our proposed methodology. In addition, we conducted two exploratory interviews. During our literature review, we identified the most common messaging protocols, such as AMQP, MQTT, Websocket, and HTTP, their respective advantages and analyzed previous work concerning interoperability between IoT protocols and standards, which were briefly explained in section 2 . In addition, we conducted exploratory, unstructured interviews with two CEOs of IoT companies. Both interview partners confirmed the need for such a tool. One partner suggested the importance of upcoming 5G technology (especially its advantages in speed and latency [19]), and as such, 5G support while preserving its benefits will be one of the defined objectives, provided the structured interviews will confirm this need in phase two.

Furthermore, we targeted the Design step by choosing the programming language Python for the development of our artifact. Python was chosen due to its capability of running on SoC-hardware as well as its general hardware independence. Additionally, Python supports multiple encryption standards, offers an extensive library of packages, and enables rapid prototyping due to its characteristic of being an interpreted programming language $[20,21]$. 
The next steps include the identification of common problems associated with IoT messaging standards selection as well as gathering additional information about the needs of practitioners through additional interviews as a baseline for our standardized questionnaire, thus completing the first step of our methodology.

\section{References}

1. Whitmore, A., Agarwal, A., Da Xu, L.: The Internet of Things-A survey of topics and trends. Information systems frontiers. 17, 261-274 (2015).

2. Braden, R.: Requirements for Internet Hosts - Communication Layers, https://tools.ietf.org/html/rfc1122, last accessed 2020/08/28.

3. Braden, R.: Requirements for Internet Hosts - Application and Support, https://tools.ietf.org/html/rfc1123, last accessed 2020/08/28.

4. Naik, N.: Choice of effective messaging protocols for IoT systems: MQTT, CoAP, AMQP and HTTP. Presented at the 2017 IEEE international systems engineering symposium (ISSE) (2017).

5. Jaikar, S.P., Iyer, K.R.: A survey of messaging protocols for IOT systems. International Journal of Advanced in Management, Technology and Engineering Sciences. 8, 510-514 (2018).

6. Peffers, K., Tuunanen, T., Rothenberger, M.A., Chatterjee, S.: A design science research methodology for information systems research. Journal of management information systems. 24, 45-77 (2007).

7. Desai, P., Sheth, A., Anantharam, P.: Semantic gateway as a service architecture for iot interoperability. Presented at the 2015 IEEE International Conference on Mobile Services (2015).

8. Iglesias-Urkia, M., Urbieta, A., Parra, J., Casado-Mansilla, D.: IEC 61850 meets CoAP: Towards the integration of Smart Grids and IoT standards. Presented at the Proceedings of the Seventh International Conference on the Internet of Things (2017).

9. Kovacs, E., Bauer, M., Kim, J., Yun, J., Le Gall, F., Zhao, M.: Standards-based worldwide semantic interoperability for IoT. IEEE Communications Magazine. 54, 40-46 (2016).

10. Yun, J., Ahn, I.-Y., Song, J., Kim, J.: Implementation of Sensing and Actuation Capabilities for IoT Devices Using oneM2M Platforms. Sensors. 19, 4567 (2019).

11. Zarko, I.P., Soursos, S., Gojmerac, I., Ostermann, E.G., Insolvibile, G., Plociennik, M., Reichl, P., Bianchi, G.: Towards an IoT framework for semantic and organizational interoperability. Presented at the 2017 Global Internet of Things Summit (GIoTS) (2017).

12. Roth, F.M., Becker, C., Vega, G., Lalanda, P.: XWARE-a customizable interoperability framework for pervasive computing systems. Pervasive and mobile computing. 47, 13-30 (2018).

13. Bandyopadhyay, S., Balamuralidhar, P., Pal, A.: Interoperation among IoT standards. Journal of ICT Standardization. 1, 253-270 (2013).

14. Rondón, R., Gidlund, M., Landernäs, K.: Evaluating bluetooth low energy suitability for time-critical industrial iot applications. International Journal of Wireless Information Networks. 24, 278-290 (2017).

15. Hevner, A.R., March, S.T., Park, J., Ram, S.: Design science in information systems research. MIS quarterly. 75-105 (2004).

16. Bell, E., Bryman, A., Harley, B.: Business research methods. Oxford university press (2018).

17. Kuzel, A.J.: Sampling in qualitative inquiry. In: Doing qualitative research. pp. 31-44. Sage Publications, Inc, Thousand Oaks, CA, US (1992). 
18. Saunders, M.N.K., Lewis, P., Thornhill, A.: Research methods for business students. Pearson, Harlow, England; New York (2012).

19. Li, S., Da Xu, L., Zhao, S.: 5G Internet of Things: A survey. Journal of Industrial Information Integration. 10, 1-9 (2018).

20. Kermarrec, F., Bourdeauducq, S., Badier, H., Le Lann, J.-C.: LiteX: an open-source SoC builder and library based on Migen Python DSL. Presented at the OSDA 2019, colocated with DATE 2019 Design Automation and Test in Europe (2019).

21. Logaras, E., Manolakos, E.S.: SysPy: using Python for processor-centric SoC design. Presented at the 2010 17th IEEE International Conference on Electronics, Circuits and Systems (2010). 